\title{
Detection of the olfactory receptor OR6V1 in cutaneous cell types in vitro and in human skin ex vivo
}

Paraskevi Gkogkolou', Mara Apel'1, Heba Dosoki', Thomas A. Luger'1, Mary Matsui², Markus Böhm'

\section{'Dept. of Dermatology, University of Münster, Münster, Germany} ${ }^{2}$ Estee Lauder, Melville, USA

\section{Introduction}

Olfactory receptors (ORs) are typically expressed in the nasal epithelium where they mediate communication between environmental odorants and the nervous system. Interestingly, there is recent evidence that expression of these $G$ protein-coupled receptors occurs also outside olfactory sensory neurons, e. g. in the skin pointing towards a broader function of ORs far beyond smell perception.

Recently, we could show that a functional OR2AT4 is expressed in human epidermal keratinocytes. A synthetic sandalwood odorant modulated the phosphorylation of mitogen-activated protein kinases in normal human keratinocytes and induced wound-healing processes in human keratinocytes via OR2AT4 (Busse et al. Invest Dermatol 2014; 134: 2823-2832).

Our aim in this study was to analyze the expression of additional ORs in three principal skin cell types in vitro as well as ex vivo in UV-exposed and non-UV-exposed forearm from healthy donors. Our findings identify the presence of another OR, OR6V1 is human skin as well in key cutaneous cell types.

\section{Results}

$\begin{array}{cccc}\begin{array}{c}\text { Target } \\ \text { gene }\end{array} & \begin{array}{c}\text { NHKs, neonatal } \\ \text { from pooled } \\ \text { donors }\end{array} & \begin{array}{c}\text { HDFs, } \\ \text { neonatal } \\ (n=3)\end{array} & \begin{array}{c}\text { NHMs, } \\ \text { neonatal } \\ (n=3)\end{array} \\ \text { OR6M1 } & + & (+)(1 / 3) & (+)(1 / 3)\end{array}$

OR11A1

OR5V1

OR6V1

$++(1 / 3)-(+),+,++(3 / 3)$

OR14A2

(OR5AX1)

OR5D16

OR6Y1

OR10J5

OR1G1

(Or17-209)

Table 1: Analysis of mRNA expression of different ORs in cultured human skin cell types in vitro by semiquantitative RT-PCR. NHKs, normal human keratinocytes; HDFs, human dermal fibroblasts; NHMs, normal human melanocytes.

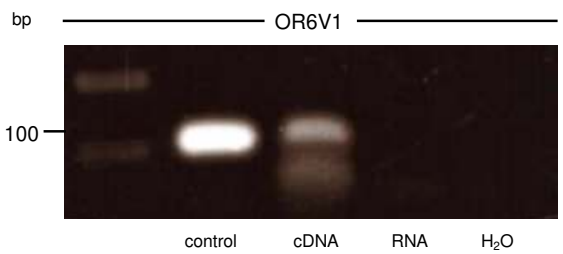

Figure 1: Expression of OR6V1 in epidermal NHKs (from 3 pooled donors) bei semi-quantitative RT-PCR analysis. Genomic DNA from diploid human cells was used as positive control and $\mathrm{H}_{2} \mathrm{O}$ as negative control.

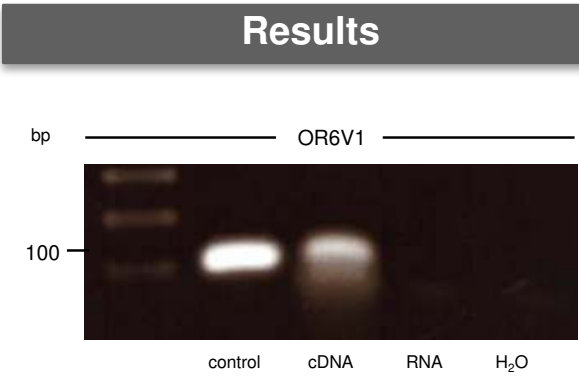

Figure 2: Expression of OR6V1 in HDFs $(n=3)$ bei RTPCR analysis. Genomic DNA from diploid human cells was used as positive control and $\mathrm{H}_{2} \mathrm{O}$ as negative control. One representative expreriment is shown.

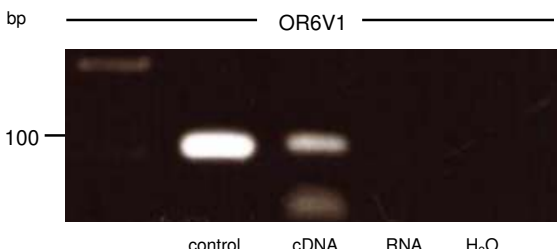

Figure 3: Expression of OR6V1 in NHMs $(n=3)$ bei RTPCR analysis. Genomic DNA from diploid human cells was used as positive control and $\mathrm{H}_{2} \mathrm{O}$ as negative control. One representative expreriment is shown.

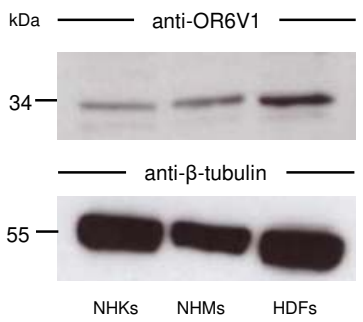

Figure 4: OR6V1 is expressed in cell lysates of cultured NHKs, NHMs and HDFs as shown by Western blot analysis.

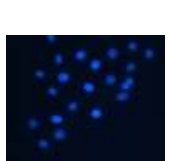

DAPI
NHKs

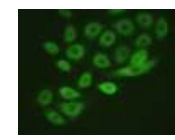

OR6V1

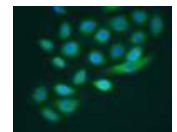

Merge
Figure 5: Expression of OR6V1 in cultured NHKs shown by immunofluorescence analysis (green fluorescence). Omission of the primary antibody did not show any staining. Nuclei were counterstained with the nucleotracker DAPI.

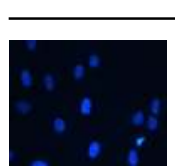

DAPI

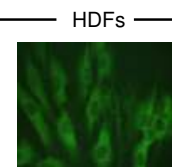

OR6V1

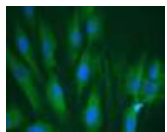

Merge
Figure 6: Expression of OR6V1 in cultured HDFs shown by immunofluorescence analysis (green fluorescence). Omission of the primary antibody did not show any staining. Nuclei were counterstained with the nucleotracker DAPI.

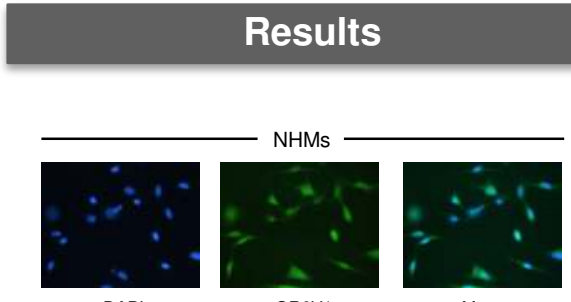

DAP

OR6V1

Merge

Figure 7: Expression of OR6V1 in cultured NHMs as shown by immunofluorescence analysis (green fluorescence). Omission of the primary antibody did not show any staining. Nuclei were counterstained with the nucleotracker DAPI.

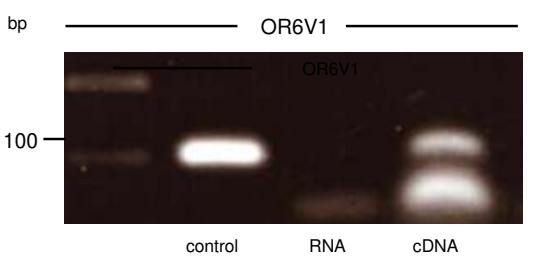

Figure 8: Expression of OR6V1 in whole skin samples from the UV-exposed forerarm of $n=10$ healthy women (age 18-35, skin phototype II-IV). DNA from diploid human cells was used as positive control. OR6V1 was found in 7 out of 10 skin samples. One representative expreriment is shown.

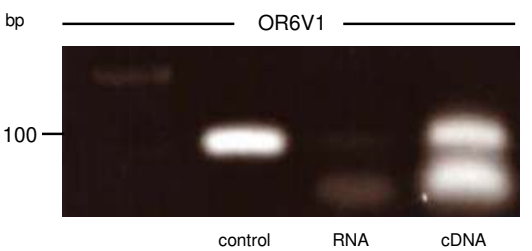

Figure 9: Expression of OR6V1 in whole skin samples from the non-UV-exposed forearm of $n=10$ healthy women (age 18-35, skin phototype II-IV). DNA from diploid human cells was used as positive control. OR6V1 was found in 8 out of 10 skin samples. One representative expreriment is shown.

\section{Conclusions}

- OR6V1 mRNA is consistently expressed in cultured $\mathrm{NHKs}$ and NHMs and variably expressed in HDFs in vitro.

- Expression of OR6V1 at the protein level was confirmed in all tested skin cell types in vitro, i. e. NHKs, HDFs and NHMs.

- Immunofluorescence staining showed a punctate cytoplasmic staining pattern of OR6V1 in all tested skin cell types.

- OR6V1 mRNA was found in the majority of UV exposed (7 out of 10) and non-UV-exposed (8 out of 10) skin samples.

- Taken together, we show for the first time the presence of a novel olfactory receptor, OR6V1, in human skin. Functional studies have to clarify the role of this receptor in cutaneous physiology and pathophysiology. 